\title{
2019 Kidney Tumor Segmentation Challenge: Medical Image Segmentation with Two-Stage Process
}

\author{
Tung-I Chen ${ }^{\star}$, Min-Sheng Wu, Yu-Cheng Chang, and Jhih-Yuan Lin \\ National Taiwan University, Computer Science Department, \\ No.1, Sec.4, Roosevelt Road, Taipei, Taiwan \\ https://www.ntu.edu.tw/english/index.html
}

\section{Introduction}

Since we are trying to deal with the medical images of real patients, the dataset are usually predominantly composed of "normal" samples. The target classes only appear in a very small portion of the entire dataset, which leads to the so-called class imbalance problem.

Besides, there is only a small percentage of foreground inside the "abnormal" images. The great majority of background leads the significant detrimental effect on training.

In such cases, model tends to focus on learning the dominant classes, leading to the poor prediction of minority class. However, the incorrect classification of pathological images can cause serious consequence in clinical practice.

\section{Methods}

The imbalanced data problem is an inevitable issue. To overcome the difficulties, our model is divided into two stages to achieve better performance.

\subsection{Classification}

The first part of our process is a two-class classification model, and we select the ResNet architecture as our model. This model is responsible for accurately classifying the image and predicting it has foreground or not. Images that has been predicted as a sample without foreground will not be sent to the segmentation stage but be transformed into background labels directly.

\subsection{Segmentation}

In the second stage, a well-training medical image segmentation model is required. According to the imbalance dataset dilemma, in this stage we decided to 


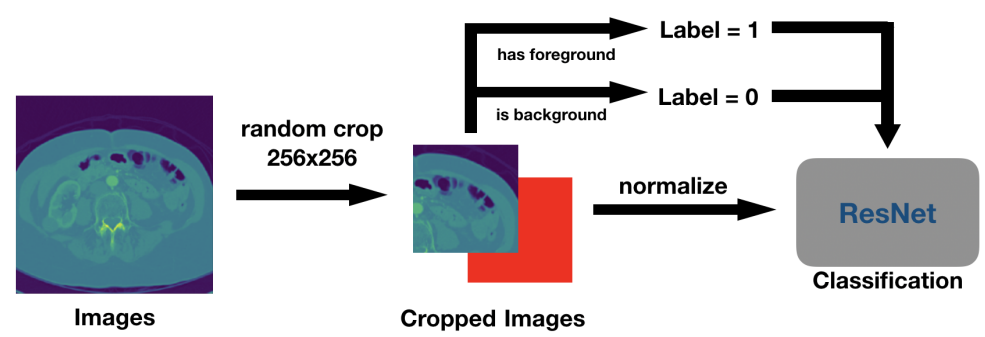

Fig. 1. The training process of classification model.

filter out the data which is only composed of background. This approach aims to refine the training dataset and makes sure the U-Net model will be forced to learn the features of foregrounds. As a result, we have a model specialized in distinguishing between kidneys and tumors in the images.

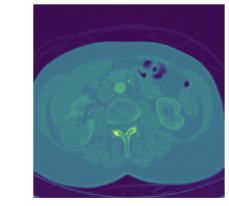

Images (have kidney or tumor)

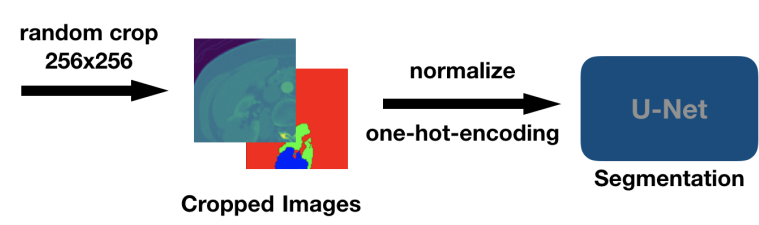

Cropped Images

Segmentation

Fig. 2. The training process of segmentation model.

\subsection{Prediction}

There are two ways to generate the outputs, and the segmentation model may not be used when predicting the testing dataset. Whether the testing data is necessary to go through the segmentation stage depends on the outputs of the classification model. Only when a image has been predicted that there is foreground inside will the image be sent to the segmentation stage. Otherwise, the output will just turn out as a zero-matrix label which indicates this image does not contain any kidney nor the tumor.

\section{Results}

Fig. 4 shows parts of the final results.

\section{Conclusion}

We have introduced a two-stage method for medical image segmentation problem. It offers an accurate segmentation for arterial phase abdominal CT scans. 


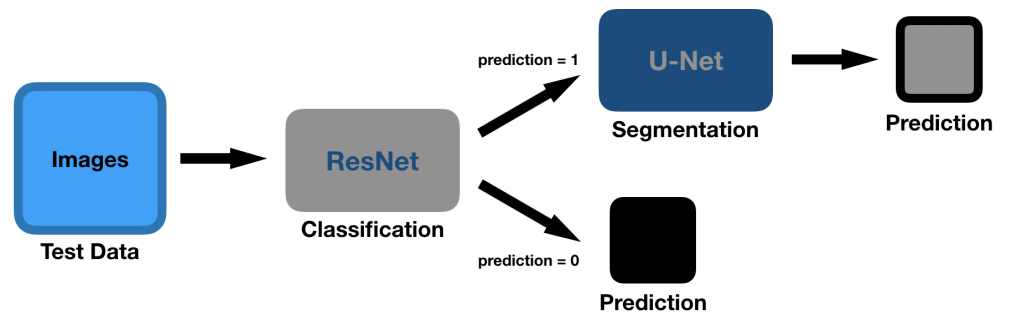

Fig. 3. The diagram of prediction.

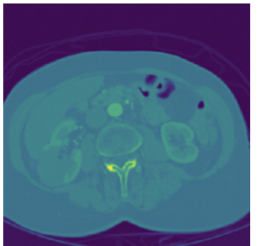

Original Image

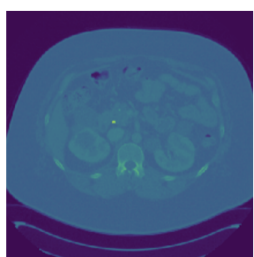

Original Image



Label

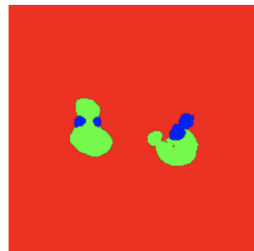

Label

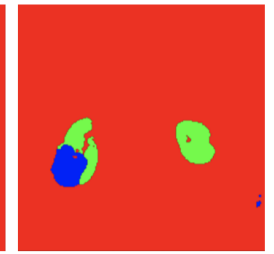

Prediction

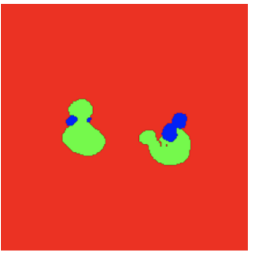

Prediction

Fig. 4. Outputs of the two-stage segmentation model. 
However, there is still room for improvement. The learning curve shows that the dice loss of the tumor is not low enough. As a result, sometimes the prediction will show the tumor but the image is actually composed of background and kidney only. We expect that it will be applicable to many other medical segmentation tasks in the future.

\section{References}

1. Kaiming He, Xiangyu Zhang, Shaoqing Ren, Jian Sun: Deep Residual Learning for Image Recognition. (2015)

2. Olaf Ronneberger, Philipp Fischer, Thomas Brox: U-Net: Convolutional Networks for Biomedical Image Segmentation. (2015) 